\title{
SET-COORDINATES FOR LATTICES ${ }^{1}$
}

\author{
ALAN D. CAMPBELL
}

On p. 26 of Garrett Birkhoff's Lattice theory (Amer. Math. Soc. Colloquium Publications, vol. 25, 1939) we find the following theorem.

THEOREM 2.12. Any partially ordered system has a one-one representation by sets which preserves inclusion and meets.

In the proof of the above theorem each element of the partially ordered system is represented by its "normal hull." We shall call this the regular representation.

Definition 1. By a representation by sets of the elements of a lattice $L$ we mean any one-one representation by sets which preserves inclusion and carries meets into set-products.

The above-mentioned regular representation is by no means the most economical. The following illustration bears out this point by using ten elements instead of the twenty-eight required by the regular representation.

ILLUSTRATION 1 . In the following manner we can represent the lattice in Figure 5 on p. 49 of Birkhoff (loc. cit.) by suitable set-coordinates (using sets of integers):

$$
\begin{aligned}
& O=(), v_{3}=(1), v_{2}=(2), v_{1}=(3), \\
& d_{1}=(1,2), d_{2}=(1,3), d_{3}=(2,3), \\
& a_{1}=(1,2,4), a_{2}=(1,3,5), d=(1,2,3), a_{3}=(2,3,6), \\
& x_{1}=(1,2,4,7), x_{2}=(1,3,5,8), e_{1}=(1,2,3,4), e_{2}=(1,2,3,5), \\
& \quad e_{3}=(1,2,3,6), x_{3}=(2,3,6,9), \\
& b_{1}=(1,2,3,4,7), b_{2}=(1,2,3,5,8), \\
& c=(1,2,3,4,5,6), b_{3}=(1,2,3,6,9), \\
& c_{1}=(1,2,3,4,5,6,7), c_{2}=(1,2,3,4,5,6,8), c_{3}=(1,2,3,4,5,6,9), \\
& u_{3}=(1,2,3,4,5,6,7,8), u_{2}=(1,2,3,4,5,6,7,9), u_{1}=(1,2,3, \\
& \quad 4,5,6,8,9), \\
& I=(1,2,3,4,5,6,7,8,9) .
\end{aligned}
$$

We remark that only nine elements (the integers $1,2,3,4,5,6,7$, $8,9)$ together with certain of the "sums" of these integers are necessary to represent this lattice (instead of the twenty-eight elements in the given Hasse diagram required in the regular representation).

Received by the editors July 25,1942 .

${ }_{1}^{1}$ The author wishes to express his gratitude to Professor Garrett Birkhoff for his advice and interest in the preparation of this paper. 
Definition 2. Ly a simple join in a lattice $L$ we mean the join of a collection $\gamma$ of elements in $L$ if this join goes by every representation by sets into the sum of the sets corresponding to the elements of $\gamma$.

ILLUSTRATION 2. Let a general finite lattice $L$ have join-irreducible elements (or atoms) $a_{1}, a_{2}, \cdots, a_{n}$. We define the set-coordinate of any $x \in L$ as the set of integers $i$ such that $a_{i} \leqq x$. Suppose that an atom 2 has above it a chain of two elements that are not joins of atoms, we can label these elements $(2, n+1)$ and $(2, n+1, n+2)$. If the atoms 3,4 , and 5 have a common simple join (that is also the join of each of the pairs 3,4 and 3,5 and 4,5$)$, then the lattice has no elements $(3,4),(3,5)$, and $(4,5)$ but has an element $(3,4,5)$.

We define the representation of Illustration 2 as the economical representation of $L$, to contrast it with the regular representation. It is an unsolved problem whether or not the economical representation actually involves the fewest possible points as set-coordinates.

Note the fact (compare Birkhoff, loc. cit., p. 26) that there exists no one-one representation of a lattice $L$ by sets which carries all meets of elements into set-products and also all joins into set-unions, unless $L$ is distributive. Also there exist representations of the elements of $L$ by sets which preserve inclusion without sending meets into set-products or joins into set-unions.

We shall denote the product (intersection) of two sets $x$ and $y$ by $x \cdot y$, the union (sum) of two sets $x$ and $y$ by $(x, y)$, and the fact that a set $z$ includes a set $w$ by $z \geqq w$. We shall denote the empty set by 0 . If two sets $u$ and $v$ have no intersection except the empty set, we shall say that $u$ and $v$ have the product 0 (in algebraic form $u \cdot v=0$ ). If the element $u$ of $L$ corresponds to the set-product $x \cdot y$, we write this fact as $u=x \cdot y$ or $u \longleftrightarrow x \cdot y$. By the expression $x-y$ we mean the set $x$ diminished by the part of the set $y$ that is included in $x$. If $x>y$ we shall say that $x$ is "over" $y$ and that $y$ is "under" $x$.

The least upper bound (or join) of any collection $\alpha$ of elements in a lattice $L$ may include elements which are not in $\alpha$, nor under $^{2} \alpha$, nor simple joins of elements in $\alpha$, nor simple joins of elements under $\alpha$. Let $\beta$ denote the set of all such elements. In this case we cannot represent the join of the elements of $\alpha$ by the sum of the sets corresponding to these elements in any representation of $L$ by sets. If in such a representation of $L$ by sets we denote by $\breve{\alpha}$ the sum of the sets corresponding to the elements of the collection $\alpha$ in $L$ (and similarly for $\beta$ ), we shall put $\breve{\beta}-\breve{\alpha}=a_{\alpha}$ (hence we have $\alpha \cdot a_{\alpha}=0$ ), and then we have the following theorem.

\footnotetext{
${ }^{2}$ By "under $\alpha$ " we mean having $\alpha$ as a set of upper bounds, see Definition 2.
} 
THEOREM 1. Suppose we have a representation of the elements of a lattice $L$ by sets. Then the meet of a collection $\alpha$ of elements of $L$ will be represented by the product of the sets of the set-union $\breve{\alpha}$ which correspond to the elements of the collection $\alpha$. The join of the elements of $\alpha$ will be represented by the set $\left(\alpha, a_{\alpha}\right)$ where the set $a_{\alpha}=\breve{\beta}-\breve{\alpha}$ and where the setunion $\breve{\beta}$ corresponds to the totality $\beta$ of elements in $L$ that are included in the join of $\alpha$ and yet are not in $\alpha$, nor under $\alpha$, nor are they simple joins of elements under $\alpha$.

Proof. The part of the proof that concerns the meet of $\alpha$ follows directly from the fact that our representation by sets carries meets into set-products. The part of the proof that concerns the join of $\alpha$ follows from the uniqueness of the join of $\alpha$, plus the uniqueness of the set $\left(\alpha, a_{\alpha}\right)$, plus the fact that the set $\left(\alpha, a_{\alpha}\right)$ is the least upper bound of the sets $\alpha$ and $\beta$, and plus the fact that our representation of elements of $L$ by sets is one-one and preserves inclusion.

Definition 3. By set-coordinates of the elements of any lattice $L$ in any representation of $L$ by sets we mean the sets corresponding to these elements.

If $x \cup y=\left(x, y, a_{x, y}\right)$ then from the paragraph on joins we see that $a_{x, y}=a_{y, x}$, also that $a_{x, y}=0$ if $y \leqq x$. Moreover $a_{x, y}=0$ if and only if $x \cup y$ contains no $z_{i}$ that have neither $x$ nor $y$ as an upper bound and that are not simple joins of elements under $x$ and $y$. The relation $x \cup(y \cup z)=(x \cup y) \cup_{z}=x \cup_{y} \cup_{z}$ implies the relation $\left(x, y, z, a_{y, z}, a_{x, y \cup z}\right)=\left(x, y, z, a_{x, y}, a_{x, y \cup z}\right)=\left(x, y, z, a_{x, y, z}\right)$ because $x \cup(y \cup z) \longleftrightarrow\left(x, y, z, a_{y, z}, a_{x, y \cup z}\right)$ and $(x \cup y) \cup z \longleftrightarrow\left(x, y, z, a_{x \cup y}\right.$, $\left.a_{x \cup y, z}\right)$ and $x \cup y \cup z \longleftrightarrow\left(x, y, z, a_{x, y, z}\right)$.

Now we shall prove some more theorems that will be of use in applying these set-coordinates to the study of lattices.

THEOREM 2. For any three elements $x, y$, and $z$ of $L$ we have (in setcoordinates) $x \cdot a_{y, z} \geqq a_{x \cdot y, x \cdot z}$.

Proof. From the latter part of Corollary 1 on p. 22 of Birkhoff (loc. cit.) we see that for any $\operatorname{such} x, y, z$ of $L$ we have $x \cap(y \cup z)$ $\geqq(x \cap y) \cup(x \cap z)$. In set-coordinates this inequality becomes $x \cdot\left(y, z, a_{y, z}\right)=\left(x \cdot y, x \cdot z, x \cdot a_{y, z}\right) \geqq\left(x \cdot y, x \cdot z, a_{x \cdot y, x}\right)$ or briefly $x \cdot a_{y, z}$ $\geqq a_{x \cdot y, x \cdot z \cdot}$

THEOREM 3. The lattice $L$ is a modular lattice if and only if for $x \geqq z$ in $L$ and for $y$ any other element of $L$ we have $x \cdot a_{y, z}=a_{x \cdot y, z}$ (in setcoordinates). 
Proof. On p. 34 of Birkhoff (loc. cit.) we find that for $L$ to be modular we must have (for $x \geqq z$ and for $y$ any other element in $L$ ) the equality $x \cap\left(y \cup_{z}\right)=(x \cap y) \cup_{z}$. In set-coordinates this equality becomes $x \cdot\left(y, z, a_{y, z}\right)=\left(x \cdot y, x \cdot z, x \cdot a_{y, z}\right)=\left(x \cdot y, z, a_{x \cdot y, z}\right)$ or finally (since $x \geqq z) x \cdot a_{y, z}=a_{x \cdot y, z}$.

ThEOREM 4. The lattice $L$ is a distributive lattice if and only if for $y$ and $z$ any two elements of $L$ we have $a_{y, z}=0$ (in set-coordinates).

Proof. On p. 74 of Birkhoff (loc. cit.) we find that one necessary and sufficient condition for $L$ to be a distributive lattice is that for all $x, y$, and $z$ in $L$ we have $x \cap(y \cup z)=(x \cap y) \cup(x \cap z)$. In setcoordinates this condition is $x \cdot\left(y, z, a_{y, z}\right)=\left(x \cdot y, x \cdot z, x \cdot a_{y, z}\right)$ $=\left(x \cdot y, x \cdot z, a_{x \cdot y, x \cdot z}\right)$ for all $x, y$, and $z$, or $x \cdot a_{y, z}=a_{x \cdot y, x \cdot z}$ or finally (since $x, y$ and $z$ are arbitrary elements of $L$ ) we have $a_{y, z}=0$ for all $y$ and $z$ in $L$.

We note that Theorem 4 asserts that a lattice is distributive if and only if every join is a simple join, and that a lattice is distributive if and only if the economical representation is join-true.

ILLUSTRATION 3. We can start in a general lattice from any set of "independent" elements and proceed as in Illustration 2 and thus obtain the part of the lattice "above" these independent elements.

ILLUSTRATION 4. The general complemented distributive lattice with $n$ atoms can readily be represented as follows. Here every join is a simple join. The atoms are $1,2, \cdots, n$. Above the atoms are the elements $(1,2),(1,3), \cdots,(n-1, n)$. Above these elements are $(1,2,3), \cdots$, and so forth up to $I=(1,2,3, \cdots, n)$.

SYRACUSE UNIVERSITY 